\title{
Bilinear equations and $q$-discrete Painlevé equations satisfied by variables and coefficients in cluster algebras
}

\author{
Naoto Okubo \\ Graduate School of Mathematical Sciences, the University of Tokyo, \\ 3-8-1 Komaba, Tokyo 153-8914, Japan
}

\begin{abstract}
We construct cluster algebras the variables and coefficients of which satisfy the discrete mKdV equation, the discrete Toda equation and other integrable bilinear equations, several of which lead to $q$ discrete Painlevé equations. These cluster algebras are obtained from quivers with an infinite number of vertices or with the mutation-period property. We will also show that a suitable transformation of quivers corresponds to a reduction of the difference equation.
\end{abstract}

\section{Introduction}

In this article, we deal with cluster algebras, which were introduced by Fomin and Zelevinsky [1, 2]. A cluster algebra is a commutative ring described by cluster variables and coefficients. A generating set of the cluster algebra is defined by mutation, which is a transformation of a seed consisting of a set of cluster variables, coefficients, and a quiver. Cluster variables and coefficients obtained from a mutation of an initial seed satisfy some difference equations. It is known that cluster variables can satisfy the discrete KdV equation [4 and the Hirota-Miwa equation [5, when the initial seed includes suitable quivers with infinite vertices [6]. These quivers have the property that an infinite number of mutations gives a permutation of its vertices. This property is called 'mutation-period' and a quiver with this property is called a mutation-periodic quiver. Several results concerning mutation-periodic quivers have been reported in [3]. All the mutation-periodic quivers in which a permutation of its vertices is achieved by a single mutation have already obtained. The quiver which gives the discrete KdV equation is obtained from a transformation of the quiver of the Hirota-Miwa equation. This transformation corresponds to a reduction from the Hirota-Miwa equation to the discrete KdV equation. In this paper, we construct the cluster algebras whose variables and coefficients satisfy the discrete mKdV equation, the discrete Toda equation [7, and some $q$-discrete Painlevé equations [8. We introduce the quiver which generalizes the one that corresponds to the discrete KdV equation and the Hirota-Miwa equation. Quivers of $q$-Painlevé I,II equations and their higher order analogues have been obtained in [6, 9. We shall introduce the quivers for the $q$-Painlevé III,VI equations, which are mutation-periodic and are obtained from transformations of quivers for the discrete $\mathrm{KdV}$ equation and the discrete $\mathrm{mKdV}$ equation.

\section{Cluster algebras}

In this section, we briefly explain the notion of cluster algebra which we use in the following sections. Let $\boldsymbol{x}=\left(x_{1}, x_{2}, \ldots, x_{N}\right), \boldsymbol{y}=\left(y_{1}, y_{2}, \ldots, y_{N}\right)$ be $N$-tuple variables. Let $Q$ be a quiver with $N$ vertices. Consider the quiver whose vertices correspond to the cluster variables. We assume that the quiver does not have a loop $(i \longrightarrow i)$ or a 2 -cycle $(i \longrightarrow j \longrightarrow i)$. Each $x_{i}$ is called a cluster variable and each $y_{i}$ is called a coefficient. The triple $(Q, \boldsymbol{x}, \boldsymbol{y})$ is called a seed. Let $\lambda_{i, j}$ be the number of arrows from $i$ to $j$ of the quiver $Q$. We define $\lambda_{i, j}$ for all $1 \leq i, j \leq N$ as $\lambda_{j, i}=-\lambda_{i, j}$. As a quiver $Q$ does not have a loop, $\lambda_{i, i}=0$. A mutation is a particular transformation of seeds.

Definition 2.1 Let $\mu_{k}:(Q, \boldsymbol{x}, \boldsymbol{y}) \longmapsto\left(Q^{\prime}, \boldsymbol{x}^{\prime}, \boldsymbol{y}^{\prime}\right) \quad(k=1,2, \ldots, N)$ be the mutation at the vertex $k$ of the quiver $Q$, defined as follows.

- $Q^{\prime}$ is a new quiver, obtained by three operations on the quiver $Q$. 
1. For all $(i, j)$ such that $\lambda_{i, k}>0, \lambda_{k, j}>0$, we add $\lambda_{i, k} \lambda_{k, j}$ arrows from $i$ to $j$.

2. If 2-cycles appear by the operation 1, we remove all of them.

3. We reverse the direction of all directed arrows which have edges at the vertex $k$.

- New coefficients $\boldsymbol{y}^{\prime}=\left(y_{1}^{\prime}, y_{2}^{\prime}, \ldots, y_{N}^{\prime}\right)$ are defined from $Q$ and $\boldsymbol{y}$ as:

$$
\begin{array}{rlrl}
y_{k}^{\prime} & =y_{k}^{-1}, & \\
y_{i}^{\prime}=y_{i}\left(y_{k}^{-1}+1\right)^{-\lambda_{k, i}} & & \left(\lambda_{k, i}>0\right), \\
y_{i}^{\prime} & =y_{i}\left(y_{k}+1\right)^{\lambda_{i, k}} & & \left(\lambda_{i, k}>0\right), \\
y_{i}^{\prime} & =y_{i} & & \left(\lambda_{k, i}=0\right) .
\end{array}
$$

- New cluster variables $\boldsymbol{x}^{\prime}=\left(x_{1}^{\prime}, x_{2}^{\prime}, \ldots, x_{N}^{\prime}\right)$ are defined from $Q, \boldsymbol{y}$ and $\boldsymbol{x}$ as:

$$
\begin{aligned}
x_{k}^{\prime} & =\frac{1}{\left(y_{k}+1\right) x_{k}}\left(\prod_{\lambda_{k, j}>0} x_{j}^{\lambda_{k, j}}+y_{k} \prod_{\lambda_{j, k}>0} x_{j}^{\lambda_{j, k}}\right), \\
x_{i}^{\prime} & =x_{i} \quad(i \neq k) .
\end{aligned}
$$

A mutation $\mu_{k}$ denotes the mutation at $k$ or $x_{k}$. For any seed $(Q, \boldsymbol{x}, \boldsymbol{y})$, it holds that $\mu_{k}^{2}(Q, \boldsymbol{x}, \boldsymbol{y})=$ $(Q, \boldsymbol{x}, \boldsymbol{y})$. For any seed $(Q, \boldsymbol{x}, \boldsymbol{y})$ and $(i, j)$ such that $\lambda_{i, j}=0$, it holds that $\mu_{i} \mu_{j}(Q, \boldsymbol{x}, \boldsymbol{y})=\mu_{j} \mu_{i}(Q, \boldsymbol{x}, \boldsymbol{y})$.

Definition 2.2 Let us fix a seed $(Q, \boldsymbol{x}, \boldsymbol{y})$. This seed is called an initial seed. Let $\mathcal{A}(Q, \boldsymbol{x}, \boldsymbol{y})$ be a cluster algebra (with coefficients) defined as

$$
\mathcal{A}(Q, \boldsymbol{x}, \boldsymbol{y})=\mathbb{Z}(\boldsymbol{y})[x \mid x \in X] \subset \mathbb{Q}(\boldsymbol{y})(\boldsymbol{x}),
$$

where $X$ is the set of all the cluster variables obtained from iterative mutations to the initial seed.

Now we define the coefficient-free cluster algebra as the pair of a quiver and cluster variables $(Q, \boldsymbol{x})$, which is also called a seed, and by using its mutation.

Definition 2.3 Let $\mu_{k}:(Q, \boldsymbol{x}) \longmapsto\left(Q^{\prime}, \boldsymbol{x}^{\prime}\right) \quad(k=1,2, \ldots, N)$ be the mutation defined as follows.

- The definition of a new quiver $Q^{\prime}$ is the same as that in Definition 2.1.

- Let $\boldsymbol{x}^{\prime}=\left(x_{1}^{\prime}, x_{2}^{\prime}, \ldots, x_{N}^{\prime}\right)$ be the new cluster variables defined by $Q$ and $\boldsymbol{x}$ as:

$$
\begin{aligned}
& x_{k}^{\prime}=\frac{1}{x_{k}}\left(\prod_{\lambda_{k, j}>0} x_{j}^{\lambda_{k, j}}+\prod_{\lambda_{j, k}>0} x_{j}^{\lambda_{j, k}}\right), \\
& x_{i}^{\prime}=x_{i} \quad(i \neq k) .
\end{aligned}
$$

Definition 2.4 Let us fix an initial seed $(Q, \boldsymbol{x})$. We define a coefficient-free cluster algebra $\mathcal{A}(Q, \boldsymbol{x})$ as

$$
\mathcal{A}(Q, \boldsymbol{x})=\mathbb{Z}[x \mid x \in X] \subset \mathbb{Q}(\boldsymbol{x}),
$$

where $X$ is the set of cluster variables obtained from a iteration of all mutations to the initial seed.

\section{Bilinear equations satisfied by cluster variables and their re- ductions}

In this section, we show that cluster variables satisfy bilinear equations related to discrete integrable systems, if the initial seed includes suitable quivers with infinite vertices. These quivers are obtained from a transformation of quivers which corresponds to a reduction of a certain difference equations. 


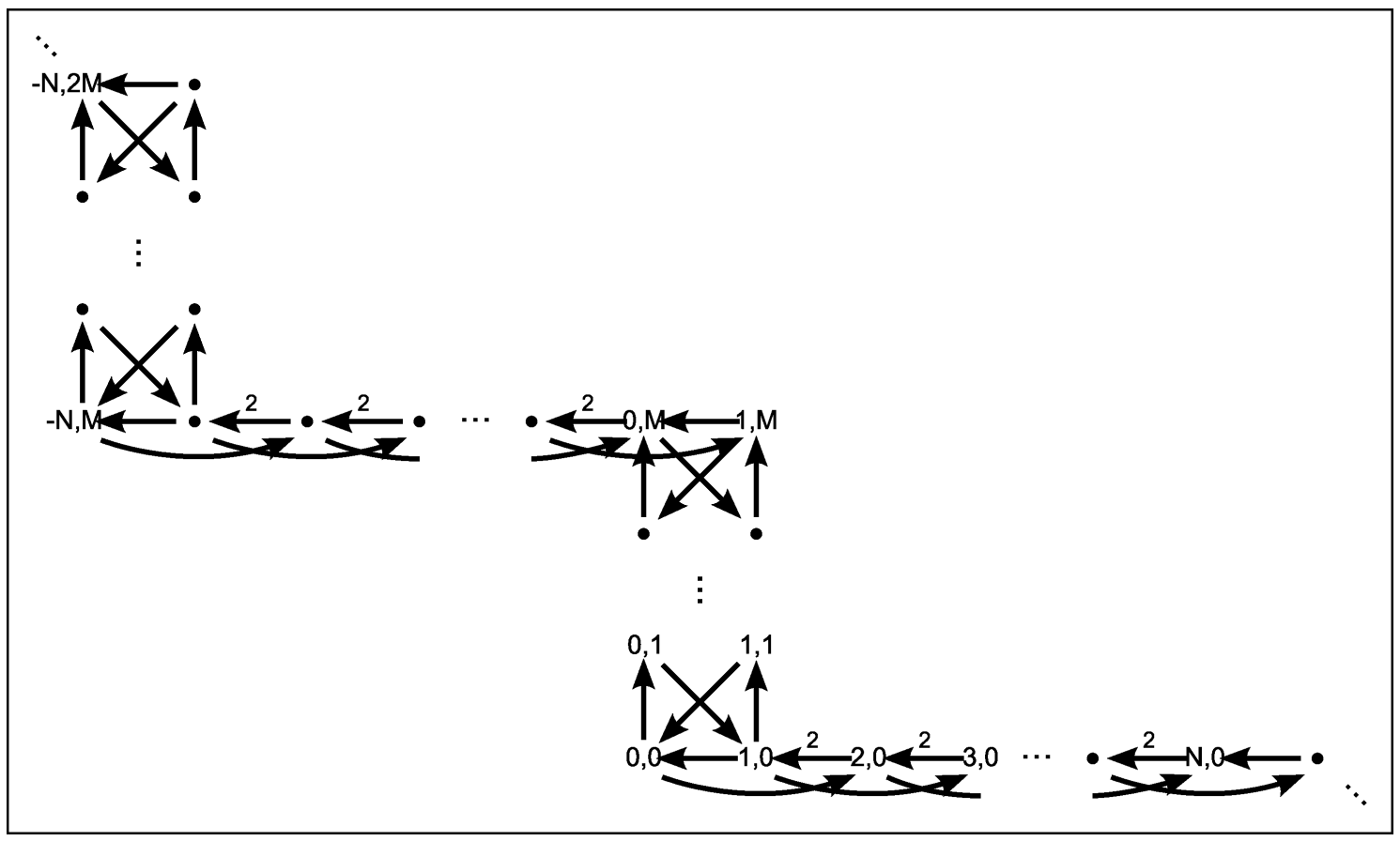

Figure 1: The dKdV quiver (The numbers on vertices correspond to cluster variables.)

\subsection{The discrete $\mathrm{KdV}$ equation and the Hirota-Miwa equation}

We construct cluster algebras whose cluster variables satisfy the discrete KdV equation and the HirotaMiwa equation. The quivers of the initial seeds were obtained in [6]. We consider quivers of general form of the discrete $\mathrm{KdV}$ equation and the Hirota-Miwa equation. First we consider coefficient-free cluster algebras. For any $N, M \geq 1$, let $Q_{\mathrm{KdV}}^{N, M}$ be a quiver as shown in Figure 1 (the dKdV quiver), where the numbers attached to the arrows of the quivers denote the numbers of arrows pointing in the same direction. Note that each vertex $(n, m)$ corresponds to a cluster variable $x_{n}^{m}$. Let $\boldsymbol{x}$ be the set of these cluster variables. We take $\left(Q_{\mathrm{KdV}}^{N, M}, \boldsymbol{x}\right)$ as an initial seed. We define $\mu_{i}^{\prime}$ as the iteration of all mutations at the vertices $i$ in Figure 2 Figure 2 shows the case where $N \geq M$. Note that mutations at vertices with the same numbers are commutative. We apply the mutation to the initial seed in the order $\mu_{1}^{\prime}, \mu_{2}^{\prime}, \ldots, \mu_{\max [N, M]}^{\prime}, \mu_{1}^{\prime}, \mu_{2}^{\prime}, \ldots$ The new cluster variable obtained by mutation at $x_{n}^{m}$ is denoted by $x_{n+2}^{m+1}$. We then obtain the following proposition from the definition of mutation (2.4).

Proposition 3.1 Consider the coefficient-free cluster algebra $\mathcal{A}\left(Q_{\mathrm{KdV}}^{N, M}, \boldsymbol{x}\right)$. For any $n, m \in \mathbb{Z}$, the cluster variables $x_{n}^{m}$ satisfy the bilinear equation

$$
x_{n+1}^{m+1} x_{n-1}^{m}=x_{n-1}^{m+1} x_{n+1}^{m}+x_{n}^{m+1} x_{n}^{m} .
$$

This equation is nothing but the bilinear form of the discrete KdV equation [4.

For any $N \geq 1$, let $Q_{\mathrm{HM}}^{N}$ be the quiver as shown in Figure 3 (the HM quiver). Vertices 1 and $N$ contained in a circle correspond to the cluster variables $x_{0,0}^{0}$ and $x_{0,-1}^{0}$ respectively, and vertices $N+1$ and 2 contained in a square correspond to the cluster variables $x_{N, 0}^{0}$ and $x_{N, 1}^{0}$ respectively. For the other vertices adjacent to $x_{n, l}^{m}$, we denote by $x_{n+1, l}^{m}$ the one on the right of $x_{n, l}^{m}$, and by $x_{n, l}^{m+1}$ the one above $x_{n, l}^{m}$, and so on. Let $\boldsymbol{x}$ be these cluster variables. We take $\left(Q_{\mathrm{HM}}^{N}, \boldsymbol{x}\right)$ as an initial seed and mutate it in the order $\mu_{1}^{\prime}, \mu_{2}^{\prime}, \ldots, \mu_{N+1}^{\prime}, \mu_{1}^{\prime}, \mu_{2}^{\prime}, \ldots$ We denote by $x_{n+1, l-1}^{m+1}$ the new cluster variable obtained by mutation at $x_{n, l}^{m}$. Then we obtain the following proposition from the definition of mutation (2.4).

Proposition 3.2 Consider the coefficient-free cluster algebra $\mathcal{A}\left(Q_{\mathrm{HM}}^{N}, \boldsymbol{x}\right)$. For any $n, m, l \in \mathbb{Z}$, the cluster variables $x_{n, l}^{m}$ satisfy the bilinear equation

$$
x_{n+1, l}^{m+1} x_{n, l+1}^{m}=x_{n, l+1}^{m+1} x_{n+1, l}^{m}+x_{n, l}^{m+1} x_{n+1, l+1}^{m} .
$$




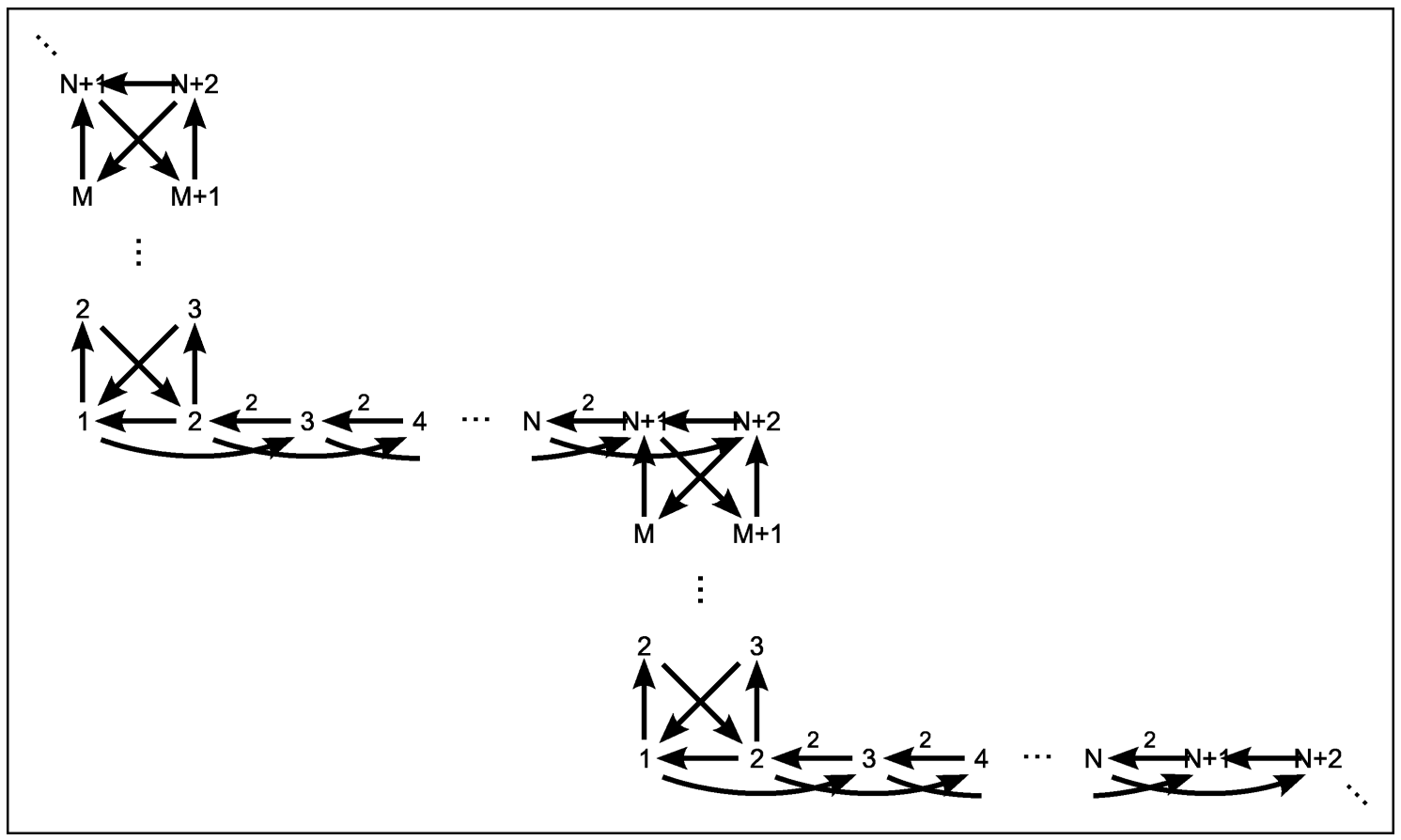

Figure 2: The dKdV quiver (The numbers at the vertices denote the order of mutations.)

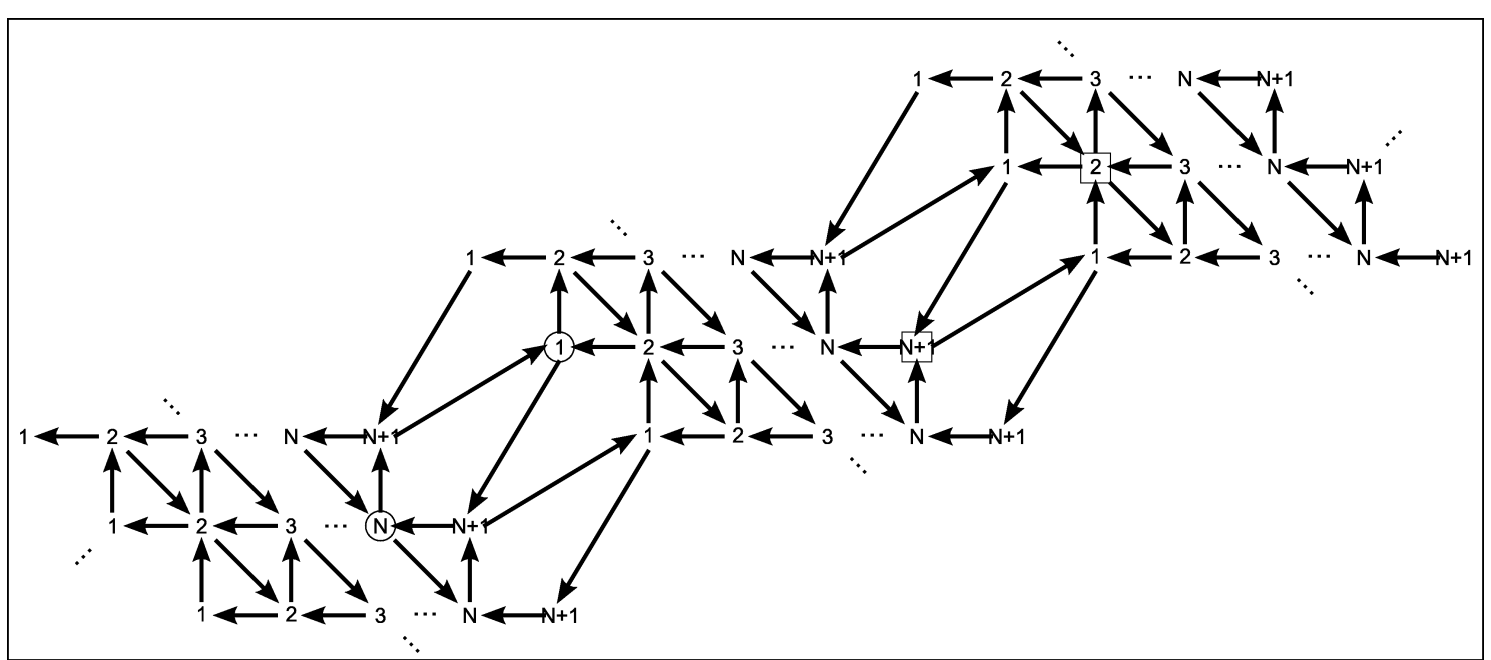

Figure 3: The HM quiver (The numbers at the vertices denote the order of mutations. Vertices 1 and $N$ contained in a circle correspond to the cluster variables $x_{0,0}^{0}$ and $x_{0,-1}^{0}$ respectively, and vertices $N+1$ and 2 contained in a square correspond to the cluster variables $x_{N, 0}^{0}$ and $x_{N, 1}^{0}$ respectively.) 


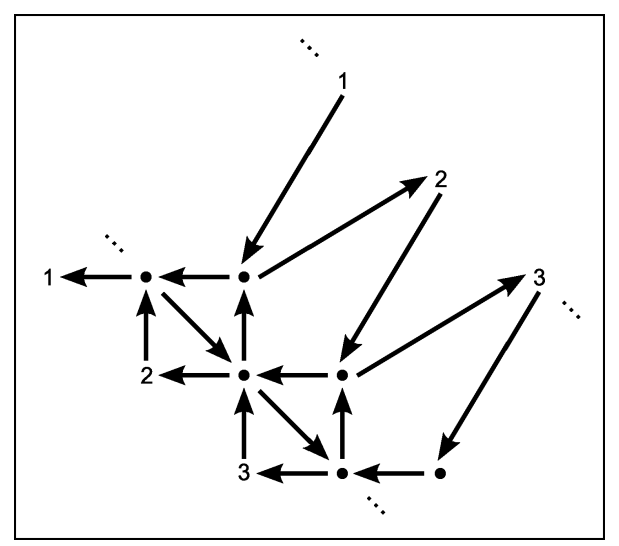

Figure 4: Reduction from the HM quiver to the $\mathrm{dKdV}$ quiver (Superposition of vertices with same numbers.)

This equation is the Hirota-Miwa equation [5].

The quiver of the discrete $\mathrm{KdV}$ equation can be obtained from a certain transformation of the quiver of the Hirota-Miwa equation. In particular, we show that the $\operatorname{dKdV}$ quiver $Q_{\mathrm{KdV}}^{1,1}$ is obtained from the HM quiver $Q_{\mathrm{HM}}^{2}$ by applying the following two operations successively on the quiver $Q_{\mathrm{HM}}^{2}$.

1. Among the arrows from $(n, m, l)+k(1,0,1)$ to $\left(n^{\prime}, m^{\prime}, l^{\prime}\right)+k(1,0,1)(k \in \mathbb{Z})$, we remove all the arrows with $k \neq 0$ in $Q_{\mathrm{HM}}^{2}$ (cf. Figure 4).

2. We superimpose the vertices $(n, m, l)+k(1,0,1)(k \in \mathbb{Z})$ on the vertex $(n, m, l)$. (In Figure 4, we superimpose the vertices with the same number.)

The $\mathrm{dKdV}$ quiver $Q_{\mathrm{KdV}}^{1,1}$ is obtained from the above operation, which will be called a $(1,0,1)$-reduction of a quiver. In a similar way the $(a, b, c)$-reduction of a quiver is defined. In fact, reduction of a quiver corresponds to reduction of a difference equation. In this case, the discrete KdV equation (3.1) is obtained from the Hirota-Miwa equation (3.2) by imposing the reduction condition $x_{n+1, l+1}^{m}=x_{n, l}^{m}$ and $x_{n}^{m}:=x_{n, 0}^{m}$.

\subsection{The discrete $\mathrm{mKdV}$ equation and the discrete Toda equation}

We construct cluster algebras whose cluster variables satisfy the discrete mKdV equation and the discrete Toda equation by the reduction of the quiver of the Hirota-Miwa equation. Let $Q_{\mathrm{mKdV}}$ (the $\mathrm{dmKdV}$ quiver) be the quiver obtained from the $(0,0,2)$-reduction of the HM quiver $Q_{\mathrm{HM}}^{1}$ (cf. Figures 5, 6). In Figure 6] the numbers at vertices denote the order of mutations. Vertices 1 in a circle and a square correspond to the cluster variables $w_{0}^{0}$ and $x_{0}^{0}$ respectively. For the other vertices adjacent to $w_{n}^{m}$ and $x_{n}^{m}$, we denote by $w_{n+1}^{m}$ and $x_{n+1}^{m}$ those on the right of $w_{n}^{m}$, and $x_{n}^{m}$ respectively, and by $w_{n}^{m+1}$, and $x_{n}^{m+1}$ those above $w_{n}^{m}$ and $x_{n}^{m}$, and so on. Let $\boldsymbol{x}$ be these cluster variables. We take $\left(Q_{\mathrm{mKdV}}, \boldsymbol{x}\right)$ as an initial seed and mutate it in the order $\mu_{1}^{\prime}, \mu_{2}^{\prime}, \mu_{1}^{\prime}, \mu_{2}^{\prime}, \ldots$ We denote by $x_{n+1}^{m+1}$ and $w_{n+1}^{m+1}$ the new cluster variable obtained by mutation at $w_{n}^{m}$ and $x_{n}^{m}$ respectively. Then we obtain the following proposition by the definition of mutation (2.4).

Proposition 3.3 Consider the coefficient-free cluster algebra $\mathcal{A}\left(Q_{\mathrm{mKdV}}, \boldsymbol{x}\right)$. For any $n, m \in \mathbb{Z}$, the cluster variables $w_{n}^{m}, x_{n}^{m}$ satisfy the bilinear equations:

$$
\begin{aligned}
& w_{n+1}^{m+1} x_{n}^{m}=x_{n}^{m+1} w_{n+1}^{m}+w_{n}^{m+1} x_{n+1}^{m}, \\
& x_{n+1}^{m+1} w_{n}^{m}=w_{n}^{m+1} x_{n+1}^{m}+x_{n}^{m+1} w_{n+1}^{m} .
\end{aligned}
$$

Note that nonautonomous bilinear equations can be obtained from cluster algebras with coefficients. Consider the cluster algebra with coefficients $\mathcal{A}\left(Q_{\mathrm{mKdV}}, \boldsymbol{x}, \boldsymbol{y}\right)$. Let the cluster variables $w_{n}^{m}, x_{n}^{m}$ be defined as in the coefficient-free case. By the definition of a mutation (2.2), the cluster variables $w_{n}^{m}, x_{n}^{m}(n, m \in \mathbb{Z})$ 


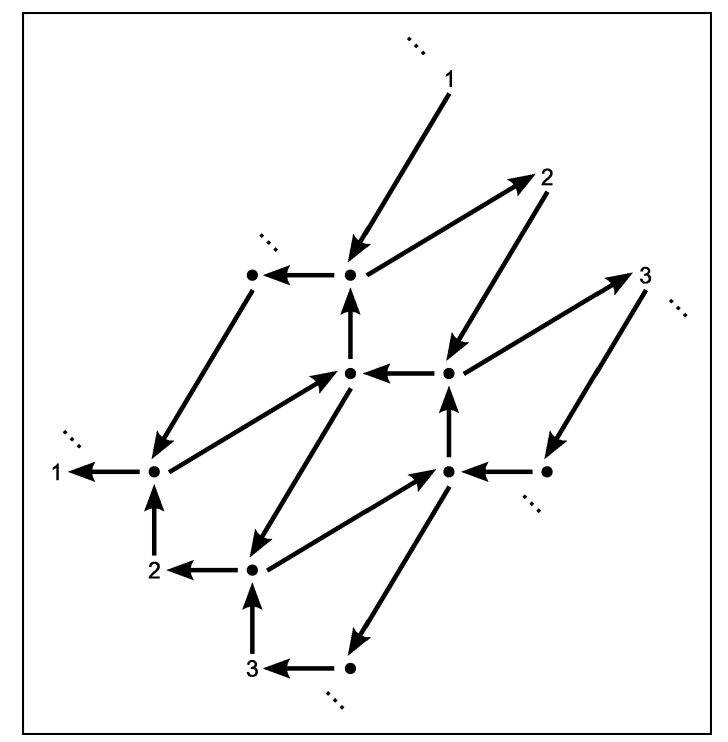

Figure 5: Reduction from the HM quiver to the dmKdV quiver

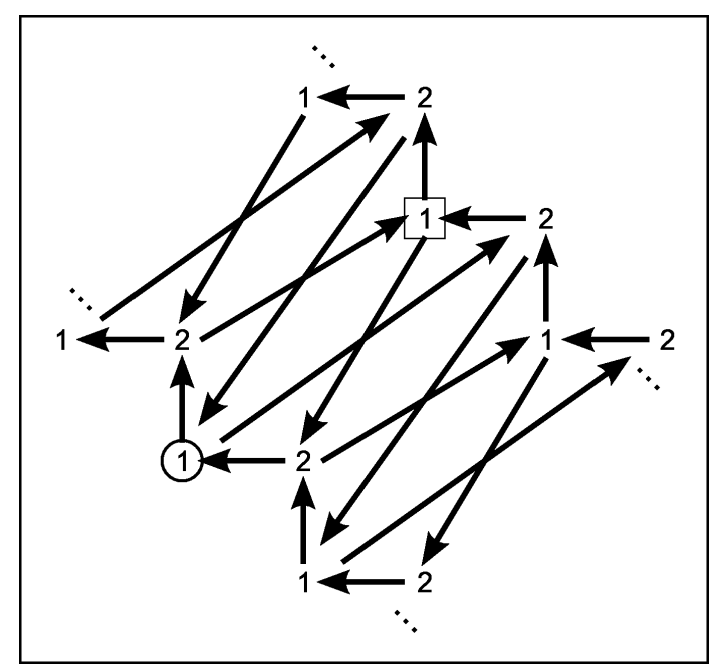

Figure 6: The dmKdV quiver

satisfy the following bilinear equations:

$$
\begin{aligned}
& w_{n+1}^{m+1} x_{n}^{m}=a_{n}^{m} x_{n}^{m+1} w_{n+1}^{m}+b_{n}^{m} w_{n}^{m+1} x_{n+1}^{m}, \\
& x_{n+1}^{m+1} w_{n}^{m}=c_{n}^{m} w_{n}^{m+1} x_{n+1}^{m}+d_{n}^{m} x_{n}^{m+1} w_{n+1}^{m},
\end{aligned}
$$

where $a_{n}^{m}, b_{n}^{m}, c_{n}^{m}, d_{n}^{m}$ are rational functions of the coefficients of the initial seed for which $a_{n}^{m}+b_{n}^{m}=$ $c_{n}^{m}+d_{n}^{m}=1$ holds. These equations are the bilinear form of the discrete mKdV equation.

The discrete $\mathrm{mKdV}$ equation (3.3) is obtained from the Hirota-Miwa equation (3.2) by imposing the reduction condition $x_{n, l+2}^{m}=x_{n, l}^{m}$ and $w_{n}^{m}:=x_{n, 0}^{m}, x_{n}^{m}:=x_{n, 1}^{m}$. This reduction of the difference equation corresponds to the $(0,0,2)$-reduction of the HM quiver. Let $Q_{\mathrm{mKdV}}^{N, M}$ be the quiver as shown in Figure 7. which shows the case of $N \geq M$. This quiver is a generalization of the dmKdV quiver. In fact, $Q_{\mathrm{mKdV}}=Q_{\mathrm{mKdV}}^{1,1}$. In Figure 7, numbers at vertices denote the order of mutations. The discrete $\mathrm{mKdV}$ equations (3.3) and (3.4) are obtained from the generalized dmKdV quiver in the same way.

Let $Q_{\mathrm{T}}$ (the dToda quiver) be the quiver obtained from the $(1,-1,1)$-reduction of the HM quiver $Q_{\mathrm{HM}}^{1}$ (cf. Figures 8, 9). In Figure 9, the numbers at vertices denote the order of mutations. The vertex 1 contained in a circle corresponds to the cluster variable $x_{0}^{0}$. For the other vertices adjacent to $x_{n}^{m}$, we denote by $x_{n+1}^{m}$ the one on the right of $x_{n}^{m}$, and by $x_{n}^{m+1}$ that above $x_{n}^{m}$, and so on. Let $\boldsymbol{x}$ be these cluster variables. We take $\left(Q_{\mathrm{T}}, \boldsymbol{x}\right)$ as an initial seed and mutate it in the order $\mu_{1}^{\prime}, \mu_{2}^{\prime}, \mu_{1}^{\prime}, \mu_{2}^{\prime}, \ldots$ We denote by $x_{n+2}^{m}$ the new cluster variable obtained by mutation at $x_{n}^{m}$. Then we obtain the following proposition by the definition of mutation (2.4).

Proposition 3.4 Consider the coefficient-free cluster algebra $\mathcal{A}\left(Q_{\mathrm{T}}, \boldsymbol{x}\right)$. For any $n, m \in \mathbb{Z}$, the cluster variables $x_{n}^{m}$ satisfy the bilinear equation

$$
x_{n+1}^{m} x_{n-1}^{m}=x_{n-1}^{m+1} x_{n+1}^{m-1}+\left(x_{n}^{m}\right)^{2} .
$$

This equation is the bilinear form of the discrete Toda equation. The discrete Toda equation (3.5) is obtained from the Hirota-Miwa equation (3.2) by imposing the reduction condition $x_{n+1, l+1}^{m-1}=x_{n, l}^{m}$ and $x_{n}^{m}:=x_{n, 0}^{m}$. This reduction of the difference equation corresponds to the $(1,-1,1)$-reduction of the HM quiver.

\section{$4 q$-discrete Painlevé equations satisfied by coefficients}

In this section, we show that coefficients in cluster algebras can satisfy $q$-discrete Painlevé equations, if the initial seed includes suitable quivers with the mutation-period property. Quivers for the $q$-Painlevé 


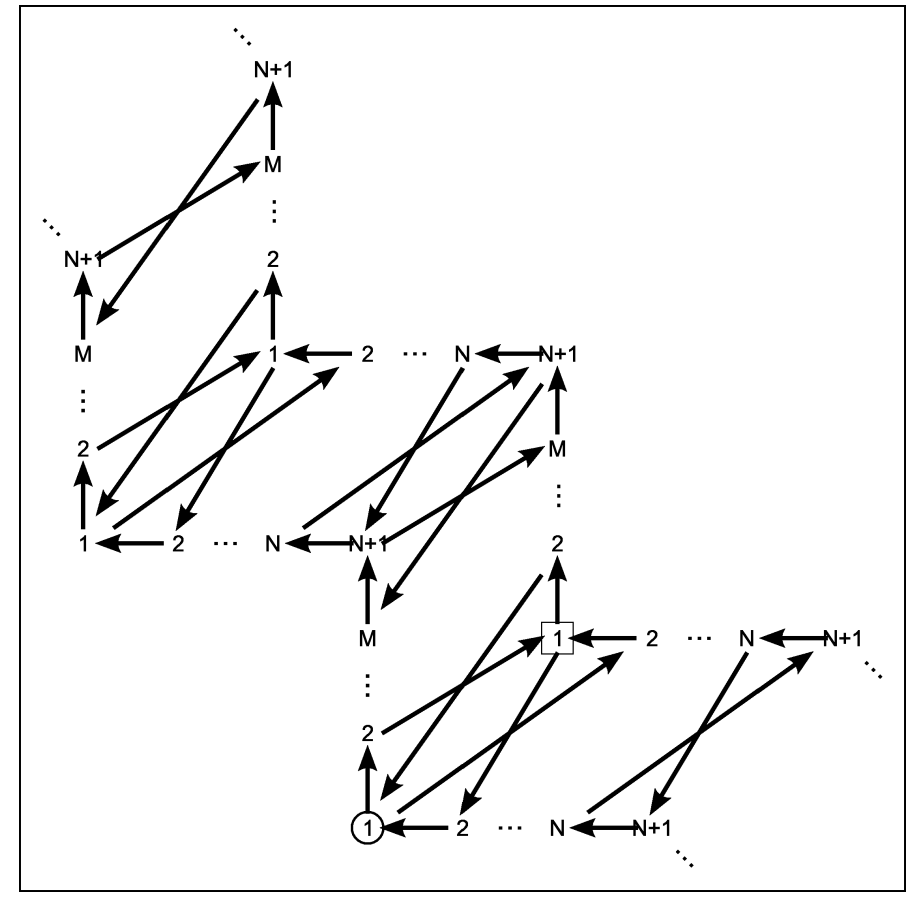

Figure 7: Generalized dmKdV quiver

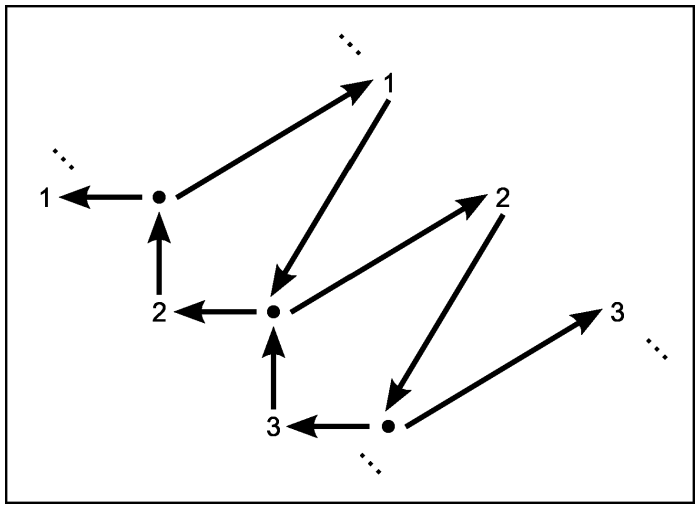

Figure 8: Reduction from the HM quiver to the dToda quiver

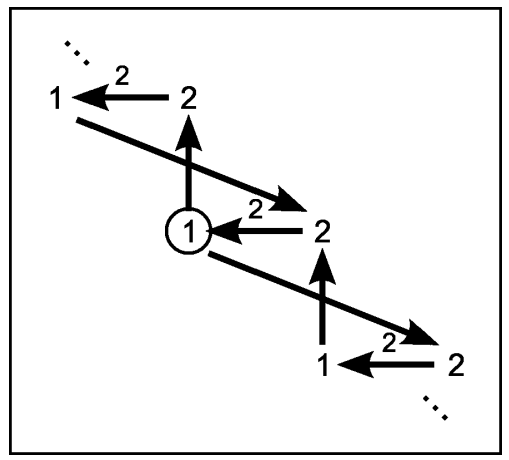

Figure 9: The dToda quiver 


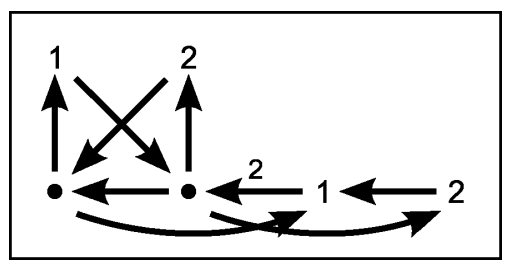

Figure 10: Reduction from the dKdV quiver to the $q$-PI quiver

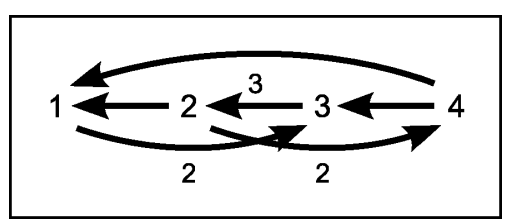

Figure 11: The $q$-PI quiver

I,II equations have been obtained in [6, 9]. In this paper, we introduce the quivers for the $q$-Painlevé III,VI equations in a similar way. We shall show that these quivers are obtained as reductions of the $\mathrm{dKdV}$ quiver and the dmKdV quiver.

\subsection{Mutation-periodic quivers}

The quivers of $q$-discrete Painlevé equations have the property that mutations of their quivers are equal to permutation of the vertices. This is the so-called 'mutation-period' property, which we define as follows. Let $Q$ be a quiver. For $\boldsymbol{i}=\left(i_{1}, i_{2}, \ldots, i_{h}\right)\left(i_{j} \in\{1,2, \ldots, N\}\right)$, we define an iteration of mutations $\mu_{\boldsymbol{i}}$ as $\mu_{\boldsymbol{i}}(Q)=\mu_{i_{h}} \mu_{i_{h-1}} \cdots \mu_{i_{1}}(Q)$, where $h$ is the number of applications of the mutation. For a permutation $\nu \in S_{N}$, let $\nu(Q)$ be the quiver in which we substituted vertices $i$ for $\nu(i)$ in $Q$.

Definition 4.1 i is a $\nu$-period of $Q$ if $\mu_{\boldsymbol{i}}(Q)=\nu(Q)$ holds. $Q$ is said to be a mutation-periodic quiver if $\boldsymbol{i}$ and $\nu \in S_{N}$, as defined above, exist.

In the case of $h=1\left(\boldsymbol{i}=\left(i_{1}\right)\right)$, all mutation-periodic quivers have already been obtained [3]. Quivers of $q$-discrete Painlevé equations are mutation-periodic quivers. These quivers arise from a reduction of the $\mathrm{dKdV}$ quiver and the $\mathrm{dmKdV}$ quiver. We consider both cluster algebras with coefficients and coefficient-free cluster algebras.

\subsection{The $q$-Painlevé I equation}

We construct the quiver of the $q$-Painlevé I equation by a reduction of the dKdV quiver. Let $Q_{\mathrm{PI}}$ (the $q$-PI quiver) be the quiver obtained from the $(2,-1)$-reduction of the $\mathrm{dKdV}$ quiver $Q_{\mathrm{KdV}}^{2,1}$ (cf. Figures 10. 11). Let $\nu \in S_{4}$ be $\nu:(1,2,3,4) \mapsto(2,3,4,1) . \quad \boldsymbol{i}=(1)$ is a $\nu$-period of $Q_{\mathrm{PI}}$. Note that each vertex $i$ corresponds to a cluster variable $x_{i}$ and a coefficient $y_{i, 1}$. We take $\left(Q_{\mathrm{PI}}, \boldsymbol{x}, \boldsymbol{y}\right)$ as an initial seed, where $\boldsymbol{x}=\left(x_{1}, x_{2}, x_{3}, x_{4}\right), \boldsymbol{y}=\left(y_{1,1}, y_{2,1}, y_{3,1}, y_{4,1}\right)$ and we mutate the initial seed in the order $\mu_{\boldsymbol{i}}=\mu_{1}, \mu_{\nu(\boldsymbol{i})}=\mu_{2}, \mu_{\nu^{2}(\boldsymbol{i})}=\mu_{3}, \ldots$ The new cluster variables and the new coefficients are denoted as $x_{n} \rightarrow x_{n+4}, y_{n, m} \rightarrow y_{n, m+1}$. We put $y_{n}:=y_{m, n}(n \equiv m(\bmod 4))$ and obtain the following seeds:

$$
\begin{aligned}
& \ldots \stackrel{\mu_{4}}{\longleftrightarrow} \quad\left(Q_{\mathrm{PI}} ; x_{1}, x_{2}, x_{3}, x_{4} ; y_{1}, y_{2,1}, y_{3,1}, y_{4,1}\right) \\
& \stackrel{\mu_{1}}{\longleftrightarrow}\left(\nu\left(Q_{\mathrm{PI}}\right) ; x_{5}, x_{2}, x_{3}, x_{4} ; y_{1,2}, y_{2}, y_{3,2}, y_{4,2}\right) \\
& \stackrel{\mu_{2}}{\longleftrightarrow}\left(\nu^{2}\left(Q_{\mathrm{PI}}\right) ; x_{5}, x_{6}, x_{3}, x_{4} ; y_{1,3}, y_{2,3}, y_{3}, y_{4,3}\right) \\
& \stackrel{\mu_{3}}{\longleftrightarrow}\left(\nu^{3}\left(Q_{\mathrm{PI}}\right) ; x_{5}, x_{6}, x_{7}, x_{4} ; y_{1,4}, y_{2,4}, y_{3,4}, y_{4}\right) \\
& \stackrel{\mu_{4}}{\longleftrightarrow} \quad\left(Q_{\mathrm{PI}} ; x_{5}, x_{6}, x_{7}, x_{8} ; y_{5}, y_{2,5}, y_{3,5}, y_{4,5}\right) \stackrel{\mu_{1}}{\longleftrightarrow} \cdots .
\end{aligned}
$$

The following proposition is obtained from the definition of mutation (2.4).

Proposition 4.2 Consider the coefficient-free cluster algebra $\mathcal{A}\left(Q_{\mathrm{PI}}, \boldsymbol{x}\right)$. For any $n \in \mathbb{Z}$, the cluster variables $x_{n}$ satisfy the bilinear equation

$$
x_{n+4} x_{n}=x_{n+2}^{2}+x_{n+3} x_{n+1} .
$$

The bilinear equation (4.2) can be obtained from the discrete $\mathrm{KdV}$ equation (3.1) by imposing the reduction condition $x_{n+2}^{m-1}=x_{n}^{m}$ and $x_{n}:=x_{n}^{0}$. This reduction of the difference equation corresponds 
to the $(2,-1)$-reduction of the $\mathrm{dKdV}$ quiver. It turns out that the corresponding coefficients satisfy the $q$-Painlevé I equation.

Theorem 4.3 Consider the cluster algebra with coefficient $\mathcal{A}\left(Q_{\mathrm{PI}}, \boldsymbol{x}, \boldsymbol{y}\right)$. For any $n \in \mathbb{Z}$, the coefficients $y_{n}$ satisfy the equation

$$
y_{n+1} y_{n-1}=c_{2} c_{1}^{n} \frac{y_{n}+1}{y_{n}^{2}},
$$

where $c_{1}, c_{2}$ are the conserved quantities

$$
c_{1}=\frac{y_{n+3}\left(y_{n+1}^{-1}+1\right)}{y_{n}\left(y_{n+2}^{-1}+1\right)}, \quad c_{2}=\frac{y_{n+2} y_{n+1}^{2} y_{n}}{y_{n+1}+1} c_{1}^{-(n+1)}
$$

and do not depend on $n$.

This equation (4.3) is the $q$-Painlevé I equation [10]. The $q$-Painlevé I equation (4.3) is obtain from the bilinear equation (4.2) by the following transformation of variables:

$$
y_{n}=\frac{x_{n+2} x_{n}}{x_{n+1}^{2}}
$$

Proof By the definition of mutation (2.1), the coefficients $y_{n, m}$ satisfy

$$
\begin{aligned}
y_{n} & =y_{n, n-1}\left(y_{n-1}+1\right), \\
y_{n, n-1} & =y_{n, n-2}\left(y_{n-2}^{-1}+1\right)^{-2}, \\
y_{n, n-2} & =y_{n, n-3}\left(y_{n-3}+1\right), \\
y_{n, n-3} & =y_{n-4}^{-1},
\end{aligned}
$$

where we consider the index $n$ of coefficients $y_{n, m}$ as $n \in \mathbb{Z} / 4 \mathbb{Z}$. We then obtain an equation only for $y_{n}$ :

$$
y_{n+4}=\frac{\left(y_{n+3}+1\right)\left(y_{n+1}+1\right)}{\left(y_{n+2}^{-1}+1\right)^{2} y_{n}} .
$$

We put

$$
u_{n}:=\frac{y_{n+3}\left(y_{n+1}^{-1}+1\right)}{y_{n}\left(y_{n+2}^{-1}+1\right)}, \quad v_{n}:=\frac{y_{n+2} y_{n+1}^{2} y_{n}}{y_{n+1}+1},
$$

and find $u_{n+1}=u_{n}$ from (4.7). Hence we obtain the conserved quantity $u_{n}=c_{1}$. Similarly, we obtain $v_{n+1}=c_{1} v_{n}$ from $u_{n}=c_{1}$ and we obtain $v_{n}=c_{2} c_{1}^{n+1}$, where $c_{2}=v_{n} c_{1}^{-(n+1)}$ is also a conserved quantity. We obtain the q-Painlevé I equation (4.3) from $v_{n}=c_{2} c_{1}^{n+1}$.

\subsection{The $q$-Painlevé II equation}

We construct the quiver of the $q$-Painlevé II equation by a reduction of dKdV quiver. Let $Q_{\text {PII }}$ (the $q$-PII quiver) be the quiver obtained from the $(3,-1)$-reduction of the $\mathrm{dKdV}$ quiver $Q_{\mathrm{KdV}}^{3,1}$ (cf. Figures 12. 13). Let $\nu \in S_{5}$ be $\nu:(1,2,3,4,5) \mapsto(2,3,4,5,1) . \boldsymbol{i}=(1)$ is a $\nu$-period of $Q_{\mathrm{PII}}$. Note that each vertex $i$ corresponds to a cluster variable $x_{i}$ and a coefficient $y_{i, 1}$. We take $\left(Q_{\mathrm{PII}}, \boldsymbol{x}, \boldsymbol{y}\right)$ as an initial seed, where $\boldsymbol{x}=\left(x_{1}, x_{2}, x_{3}, x_{4}, x_{5}\right), \boldsymbol{y}=\left(y_{1,1}, y_{2,1}, y_{3,1}, y_{4,1}, y_{5,1}\right)$ and mutate the initial seed in the order $\mu_{\boldsymbol{i}}, \mu_{\nu(\boldsymbol{i})}, \mu_{\nu^{2}(\boldsymbol{i})}, \ldots$ The new cluster variables and the new coefficients are denoted as $x_{n} \rightarrow x_{n+5}, y_{n, m} \rightarrow$ $y_{n, m+1}$. We put $y_{n}:=y_{m, n}(n \equiv m(\bmod 5))$ and obtain the following seeds:

$$
\begin{aligned}
& \cdots \stackrel{\mu_{5}}{\longrightarrow} \quad\left(Q_{\mathrm{PII}} ; x_{1}, x_{2}, x_{3}, x_{4}, x_{5} ; y_{1}, y_{2,1}, y_{3,1}, y_{4,1}, y_{5,1}\right) \\
& \stackrel{\mu_{1}}{\longleftrightarrow}\left(\nu\left(Q_{\mathrm{PII}}\right) ; x_{6}, x_{2}, x_{3}, x_{4}, x_{5} ; y_{1,2}, y_{2}, y_{3,2}, y_{4,2}, y_{5,2}\right) \\
& \stackrel{\mu_{2}}{\longleftrightarrow}\left(\nu^{2}\left(Q_{\mathrm{PII}}\right) ; x_{6}, x_{7}, x_{3}, x_{4}, x_{5} ; y_{1,3}, y_{2,3}, y_{3}, y_{4,3}, y_{5,3}\right) \stackrel{\mu_{3}}{\longleftrightarrow} \cdots .
\end{aligned}
$$

We obtain the following proposition from the definition of mutation (2.4). 


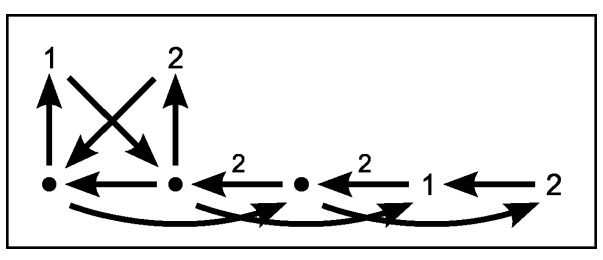

Figure 12: Reduction from the dKdV quiver to the $q$-PII quiver

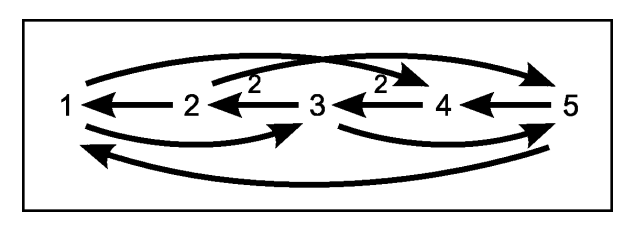

Figure 13: The $q$-PII quiver

Proposition 4.4 Consider the coefficient-free cluster algebra $\mathcal{A}\left(Q_{\mathrm{PII}}, \boldsymbol{x}\right)$. For any $n \in \mathbb{Z}$, the cluster variables $x_{n}$ satisfy the bilinear equation

$$
x_{n+5} x_{n}=x_{n+3} x_{n+2}+x_{n+4} x_{n+1} .
$$

The bilinear equation (4.10) is obtained from the discrete KdV equation (3.1) by imposing the reduction condition $x_{n+3}^{m-1}=x_{n}^{m}$ and $x_{n}:=x_{n}^{0}$. This reduction of the difference equation corresponds to the $(3,-1)$-reduction of the dKdV quiver. Its coefficients satisfy the $q$-Painlevé II equation.

Theorem 4.5 Consider the cluster algebra with coefficient $\mathcal{A}\left(Q_{\mathrm{PII}}, \boldsymbol{x}, \boldsymbol{y}\right)$. For any $n \in \mathbb{Z}$, the coefficients $y_{n}$ satisfy the equation

$$
y_{n+1} y_{n-1}=c_{2} c_{3}^{(-1)^{n}} c_{1}^{n} \frac{y_{n}+1}{y_{n}},
$$

where $c_{1}, c_{2}, c_{3}$ are the conserved quantities

$$
\begin{aligned}
& c_{1}^{2}=\frac{y_{n+4}\left(y_{n+1}^{-1}+1\right)}{y_{n}\left(y_{n+3}^{-1}+1\right)}, \quad c_{2}^{2}=\frac{y_{2 n+3} y_{2 n+2}^{2} y_{2 n+1}^{2} y_{2 n}}{\left(y_{2 n+2}+1\right)\left(y_{2 n+1}+1\right)} c_{1}^{-(4 n+3)}, \\
& c_{3}^{2}=\frac{y_{2 n+3}\left(y_{2 n+1}+1\right)}{y_{2 n}\left(y_{2 n+2}+1\right)} c_{1}^{-1}
\end{aligned}
$$

and do not depend on $n$.

We put $f_{n}:=y_{2 n}, g_{n}:=y_{2 n+1}$ and obtain

$$
\begin{aligned}
& f_{n+1} f_{n}=c_{2} c_{3}^{-1} c_{1}^{2 n+1} \frac{g_{n}+1}{g_{n}}, \\
& g_{n} g_{n-1}=c_{2} c_{3} c_{1}^{2 n} \frac{f_{n}+1}{f_{n}}
\end{aligned}
$$

from (4.11). This equation is the $q$-Painlevé II equation [11]. The $q$-Painlevé II equation (4.11) is obtained from the bilinear equation (4.10) by the following transformation of variables:

$$
y_{n}=\frac{x_{n+3} x_{n}}{x_{n+2} x_{n+1}} .
$$

Proof By the definition of mutation (2.1), the coefficients $y_{n, m}$ satisfy

$$
\begin{aligned}
y_{n} & =y_{n, n-1}\left(y_{n-1}+1\right), \\
y_{n, n-1} & =y_{n, n-2}\left(y_{n-2}^{-1}+1\right)^{-1}, \\
y_{n, n-2} & =y_{n, n-3}\left(y_{n-3}^{-1}+1\right)^{-1}, \\
y_{n, n-3} & =y_{n, n-4}\left(y_{n-4}+1\right), \\
y_{n, n-4} & =y_{n-5}^{-1},
\end{aligned}
$$

where we think of the index $n$ of the coefficients $y_{n, m}$ as $n \in \mathbb{Z} / 5 \mathbb{Z}$. We obtain an equation only for $y_{n}$ :

$$
y_{n+5}=\frac{\left(y_{n+4}+1\right)\left(y_{n+1}+1\right)}{\left(y_{n+3}^{-1}+1\right)\left(y_{n+2}^{-1}+1\right) y_{n}} .
$$




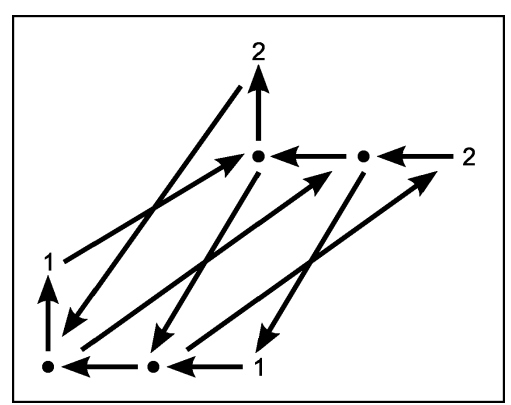

Figure 14: Reduction from the dmKdV quiver to the $q$-PIII quiver

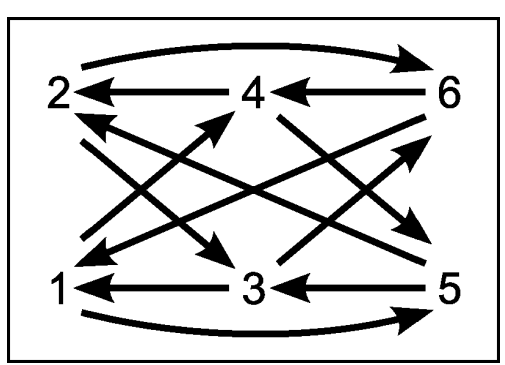

Figure 15: The $q$-PIII quiver

We put

$$
u_{n}:=\frac{y_{n+4}\left(y_{n+1}^{-1}+1\right)}{y_{n}\left(y_{n+3}^{-1}+1\right)}, \quad v_{n}:=\frac{y_{n+2} y_{n}}{y_{n+1}^{-1}+1},
$$

and find $u_{n+1}=u_{n}$ from (4.16). Hence, we obtain the conserved quantity $u_{n}=c_{1}^{2}$. Similarly, we obtain $v_{n+2}=c_{1}^{2} v_{n}$ from $u_{n}=c_{1}^{2}$, and $v_{n}=c_{2} c_{3}^{(-1)^{n+1}} c_{1}^{n+1} ; c_{2}^{2}=v_{2 n+1} v_{2 n} c_{1}^{-(4 n+3)}$ and $c_{3}^{2}=v_{2 n+1} v_{2 n}^{-1} c_{1}^{-1}$ are conserved quantities. We then obtain the q-Painlevé II equation (4.11) from $v_{n}=c_{2} c_{3}^{(-1)^{n+1}} c_{1}^{n+1}$.

\subsection{The $q$-Painlevé III equation}

We construct the quiver of the $q$-Painlevé III equation by a reduction of the dmKdV quiver. Let $Q_{\text {PIII }}$ (the $q$-PIII quiver) be the quiver obtained from the $(2,-1)$-reduction of the $\operatorname{dmKdV}$ quiver $Q_{\mathrm{mKdV}}^{2,1}$ (cf. Figures 14, 15). Let $\nu \in S_{6}$ be $\nu:(1,2,3,4,5,6) \mapsto(3,4,5,6,2,1) . \quad \boldsymbol{i}=(1,2)$ is a $\nu$-period of $Q_{\mathrm{PIII}}$. Note that vertices $(1,2,3,4,5,6)$ correspond to cluster variables $\boldsymbol{x}=\left(w_{1}, x_{1}, w_{2}, x_{2}, w_{3}, x_{3}\right)$ and coefficients $\boldsymbol{y}=\left(y_{1,1}, z_{1,1}, y_{2,1}, z_{2,1}, y_{3,1}, z_{3,1}\right)$. We take $\left(Q_{\mathrm{PIII}}, \boldsymbol{x}, \boldsymbol{y}\right)$ as an initial seed and mutate it in the order $\mu_{\boldsymbol{i}}=\mu_{2} \mu_{1}, \mu_{\nu(\boldsymbol{i})}=\mu_{4} \mu_{3}, \mu_{\nu^{2}(\boldsymbol{i})}=\mu_{6} \mu_{5}, \ldots$. The new cluster variables are denoted as $w_{n} \rightarrow x_{n+3}, x_{n} \rightarrow w_{n+3}$ and the new coefficients are denoted as $y_{n, m} \rightarrow z_{n, m+1}, z_{n, m} \rightarrow y_{n, m+1}$ (if the coefficient corresponds to the vertex to which the mutation is applied) or $y_{n, m} \rightarrow y_{n, m+1}, z_{n, m} \rightarrow z_{n, m+1}$ (otherwise). We put $y_{n}:=y_{m, n}, z_{n}:=z_{m, n}(n \equiv m(\bmod 3))$ and obtain the following seeds:

$$
\begin{aligned}
& \ldots \stackrel{\mu_{5} \mu_{6}}{\longrightarrow} \quad\left(Q_{\mathrm{PIII}} ; w_{1}, x_{1}, w_{2}, x_{2}, w_{3}, x_{3} ; y_{1}, z_{1}, y_{2,1}, z_{2,1}, y_{3,1}, z_{3,1}\right) \\
& \stackrel{\mu_{2} \mu_{7}}{\longleftrightarrow}\left(\nu\left(Q_{\mathrm{PIII}}\right) ; x_{4}, w_{4}, w_{2}, x_{2}, w_{3}, x_{3} ; z_{1,2}, y_{1,2}, y_{2}, z_{2}, y_{3,2}, z_{3,2}\right) \\
& \stackrel{\mu_{4} \mu_{3}}{\longleftrightarrow}\left(\nu^{2}\left(Q_{\mathrm{PIII}}\right) ; x_{4}, w_{4}, x_{5}, w_{5}, w_{3}, x_{3} ; z_{1,3}, y_{1,3}, z_{2,3}, y_{2,3}, y_{3}, z_{3}\right) \\
& \stackrel{\mu_{6} \mu_{5}}{\longleftrightarrow}\left(Q_{\mathrm{PIII}} ; x_{4}, w_{4}, x_{5}, w_{5}, x_{6}, w_{6} ; z_{4}, y_{4}, z_{2,4}, y_{2,4}, z_{3,4}, y_{3,4}\right) \\
& \stackrel{\mu_{1} \mu_{2}}{\longleftrightarrow}\left(\nu\left(Q_{\mathrm{III}}\right) ; w_{7}, x_{7}, x_{5}, w_{5}, x_{6}, w_{6} ; y_{1,5}, z_{1,5}, z_{5}, y_{5}, z_{3,5}, y_{3,5}\right) \stackrel{\mu_{3} \mu_{4}}{\longleftrightarrow} \ldots .
\end{aligned}
$$

We obtain the following proposition from the definition of mutation (2.4).

Proposition 4.6 Consider the coefficient-free cluster algebra $\mathcal{A}\left(Q_{\mathrm{PIII}}, \boldsymbol{x}\right)$. For any $n \in \mathbb{Z}$, the cluster variables $w_{n}, x_{n}$ satisfy the bilinear equations:

$$
\begin{aligned}
& w_{n+3} x_{n}=x_{n+2} w_{n+1}+w_{n+2} x_{n+1}, \\
& x_{n+3} w_{n}=w_{n+2} x_{n+1}+x_{n+2} w_{n+1} .
\end{aligned}
$$

The bilinear equations (4.19) are obtained from the discrete mKdV equation (3.3) by imposing the reduction condition $w_{n+2}^{m-1}=w_{n}^{m}, x_{n+2}^{m-1}=x_{n}^{m}$ and $w_{n}:=w_{n}^{0}, x_{n}:=x_{n}^{0}$. This reduction of the difference equation corresponds to the $(2,-1)$-reduction of the dmKdV quiver. In this case, the coefficients satisfy the $q$-Painlevé III equation. 
Theorem 4.7 Consider the cluster algebra with coefficient $\mathcal{A}\left(Q_{\mathrm{PIII}}, \boldsymbol{x}, \boldsymbol{y}\right)$. For any $n \in \mathbb{Z}$, the coefficients $y_{n}, z_{n}$ satisfy the equations:

$$
\begin{aligned}
& y_{n+1} y_{n-1}=c_{2} c_{3}^{2} c_{1}^{2 n} \frac{y_{n}+1}{y_{n}\left(y_{n}+c_{3} c_{4}^{(-1)^{n}} c_{1}^{n}\right)}, \\
& z_{n+1} z_{n-1}=c_{2}^{-1} c_{3}^{2} c_{1}^{2 n} \frac{z_{n}+1}{z_{n}\left(z_{n}+c_{3} c_{4}^{(-1)^{n}} c_{1}^{n}\right)},
\end{aligned}
$$

where $c_{1}, c_{2}, c_{3}, c_{4}$ are conserved quantities

$$
\begin{aligned}
c_{1}^{2} & =\frac{y_{n+2} z_{n+2}}{y_{n} z_{n}}, & c_{2}^{2} & =\frac{y_{n+2} y_{n}\left(z_{n+1}^{-1}+1\right)^{2}}{z_{n+2} z_{n}\left(y_{n+1}^{-1}+1\right)^{2}} \\
c_{3}^{2} & =y_{2 n+1} z_{2 n+1} y_{2 n} z_{2 n} c_{1}^{-(4 n+1)}, & c_{4}^{2} & =\frac{y_{2 n} z_{2 n}}{y_{2 n+1} z_{2 n+1}} c_{1}
\end{aligned}
$$

and do not depend on $n$.

If we put $f_{n}:=y_{2 n}, g_{n}:=y_{2 n+1}$, we obtain

$$
\begin{aligned}
& f_{n+1} f_{n}=c_{2} c_{3}^{2} c_{1}^{4 n+2} \frac{g_{n}+1}{g_{n}\left(g_{n}+c_{3} c_{4}^{-1} c_{1}^{2 n+1}\right)}, \\
& g_{n} g_{n-1}=c_{2} c_{3}^{2} c_{1}^{4 n} \frac{f_{n}+1}{f_{n}\left(f_{n}+c_{3} c_{4} c_{1}^{2 n}\right)}
\end{aligned}
$$

from (4.20). These equations are the $q$-Painlevé III equation [12].

Proof By the definition of mutation (2.1), the coefficients $y_{n, m}, z_{n, m}$ satisfy

$$
\begin{aligned}
y_{n} & =y_{n, n-1}\left(y_{n-1}+1\right)\left(z_{n-1}^{-1}+1\right)^{-1}, \\
y_{n, n-1} & =y_{n, n-2}\left(y_{n-2}^{-1}+1\right)^{-1}\left(z_{n-2}+1\right), \\
y_{n, n-2} & =z_{n-3}^{-1}, \\
z_{n} & =z_{n, n-1}\left(z_{n-1}+1\right)\left(y_{n-1}^{-1}+1\right)^{-1}, \\
z_{n, n-1} & =z_{n, n-2}\left(z_{n-2}^{-1}+1\right)^{-1}\left(y_{n-2}+1\right), \\
z_{n, n-2} & =y_{n-3}^{-1},
\end{aligned}
$$

where we consider the index $n$ of the coefficients $y_{n, m}, z_{n, m}$ as $n \in \mathbb{Z} / 3 \mathbb{Z}$. The equations, only for $y_{n}, z_{n}$, are

$$
\begin{aligned}
& y_{n+3}=\frac{\left(y_{n+2}+1\right)\left(z_{n+1}+1\right)}{\left(z_{n+2}^{-1}+1\right)\left(y_{n+1}^{-1}+1\right) z_{n}}, \\
& z_{n+3}=\frac{\left(z_{n+2}+1\right)\left(y_{n+1}+1\right)}{\left(y_{n+2}^{-1}+1\right)\left(z_{n+1}^{-1}+1\right) y_{n}} .
\end{aligned}
$$

If we put

$$
u_{n}:=\frac{y_{n+2}\left(z_{n+1}^{-1}+1\right)}{z_{n}\left(y_{n+1}^{-1}+1\right)}, \quad v_{n}:=\frac{z_{n+2}\left(y_{n+1}^{-1}+1\right)}{y_{n}\left(z_{n+1}^{-1}+1\right)}, \quad t_{n}:=y_{n} z_{n},
$$

we find $u_{n+1}=u_{n}, v_{n+1}=v_{n}$ from (4.24), and obtain conserved quantities $u_{n}=c_{1} c_{2}, v_{n}=c_{1} c_{2}^{-1}$. We also obtain $t_{n+2}=c_{1}^{2} t_{n}$ from $u_{n} v_{n}=c_{1}^{2}$, and $t_{n}=c_{3} c_{4}^{(-1)^{n}} c_{1}^{n} \cdot c_{3}^{2}=t_{2 n+1} t_{2 n} c_{1}^{-(4 n+1)}$ and $c_{4}^{2}=t_{2 n+1}^{-1} t_{2 n} c_{1}$ are conserved quantities. We obtain the q-Painlevé III equation (4.20) from $u_{n}=c_{1} c_{2}$ and $t_{n}=c_{3} c_{4}^{(-1)^{n}} c_{1}^{n}$ by the elimination of $z_{n}$. The equation for $z_{n}$ is the same as that for $y_{n}$. 


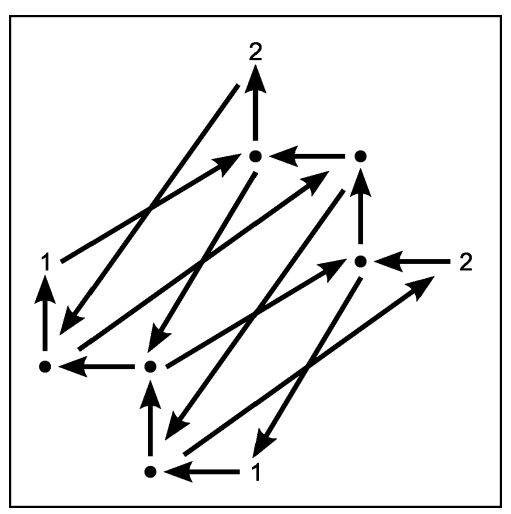

Figure 16: Reduction from the dmKdV quiver to the $q$-PVI quiver

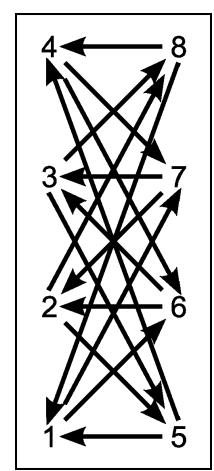

Figure 17: The $q$-PVI quiver

\subsection{The $q$-Painlevé VI equation}

We now construct the quiver of the $q$-Painlevé VI equation by a reduction of the $\operatorname{dmKdV}$ quiver. Let $Q_{\mathrm{PVI}}$ (the $q$-PVI quiver) be the quiver obtained from the $(2,-2)$-reduction of the $\operatorname{dmKdV}$ quiver $Q_{\mathrm{mKdV}}^{1,1}$ (cf. Figures 16, 17). Let $\nu \in S_{8}$ be $\nu:(1,2,3,4,5,6,7,8) \mapsto(5,6,7,8,4,3,2,1) . \boldsymbol{i}=(1,2,3,4)$ is a $\nu$-period of $Q_{\mathrm{PVI}}$. Note that vertices $(1,2, \ldots, 8)$ correspond to cluster variables $\boldsymbol{x}=\left(w_{1}, x_{1}, W_{1}, X_{1}, w_{2}, x_{2}, W_{2}, X_{2}\right)$ and coefficients $\boldsymbol{y}=\left(y_{1,1}, z_{1,1}, Y_{1,1}, Z_{1,1}, y_{2,1}, z_{2,1}, Y_{2,1}, Z_{2,1}\right)$. We take $\left(Q_{\mathrm{PVI}}, \boldsymbol{x}, \boldsymbol{y}\right)$ as an initial seed and mutate it in the order $\mu_{\boldsymbol{i}}, \mu_{\nu(\boldsymbol{i})}, \mu_{\nu^{2}(\boldsymbol{i})}, \ldots$ The new cluster variables are denoted as $w_{n} \rightarrow X_{n+2}, x_{n} \rightarrow$ $W_{n+2}, W_{n} \rightarrow x_{n+2}, Z_{n} \rightarrow w_{n+2}$ and the new coefficients are denoted as $y_{n, m} \rightarrow Z_{n, m+1}, z_{n, m} \rightarrow$ $Y_{n, m+1}, Y_{n, m} \rightarrow z_{n, m+1}, Z_{n, m} \rightarrow y_{n, m+1}$ (in case the coefficient corresponds to the vertex to which the mutation is applied) or $y_{n, m} \rightarrow y_{n, m+1}, z_{n, m} \rightarrow z_{n, m+1}, Y_{n, m} \rightarrow Y_{n, m+1}, Z_{n, m} \rightarrow Z_{n, m+1}$ (otherwise). We put $y_{n}:=y_{m, n}, z_{n}:=z_{m, n}, Y_{n}:=Y_{m, n}, Z_{n}:=Z_{m, n}(n \equiv m(\bmod 2))$ and obtain the following seeds:

$$
\begin{aligned}
& \ldots \stackrel{\mu_{5} \mu_{6} \mu_{7} \mu_{8}}{\longleftrightarrow}\left(Q_{\mathrm{VI}} ; w_{1}, x_{1}, W_{1}, X_{1}, w_{2}, x_{2}, W_{2}, X_{2} ;\right. \\
& \left.y_{1}, z_{1}, Y_{1}, Z_{1}, y_{2,1}, z_{2,1}, Y_{2,1}, Z_{2,1}\right) \\
& \stackrel{\mu_{4} \mu_{3} \mu_{2} \mu_{1}}{\longleftrightarrow}\left(\nu\left(Q_{\mathrm{VI}}\right) ; X_{3}, W_{3}, x_{3}, w_{3}, w_{2}, x_{2}, W_{2}, X_{2}\right. \text {; } \\
& \left.Z_{1,2}, Y_{1,2}, z_{1,2}, y_{1,2}, y_{2}, z_{2}, Y_{2}, Z_{2}\right) \\
& \stackrel{\mu_{8} \mu_{7} \mu_{6} \mu_{5}}{\longrightarrow}\left(Q_{\mathrm{VI}} ; X_{3}, W_{3}, x_{3}, w_{3}, X_{4}, W_{4}, x_{4}, w_{4}\right. \text {; } \\
& \left.Z_{3}, Y_{3}, z_{3}, y_{3}, Z_{2,3}, Y_{2,3}, z_{2,3}, y_{2,3}\right) \\
& \stackrel{\mu_{1} \mu_{2} \mu_{3} \mu_{4}}{\longleftrightarrow}\left(\nu\left(Q_{\mathrm{VI}}\right) ; w_{5}, x_{5}, W_{5}, X_{5}, X_{4}, W_{4}, x_{4}, w_{4}\right. \text {; } \\
& \left.y_{1,4}, z_{1,4}, Y_{1,4}, Z_{1,4}, Z_{4}, Y_{4}, z_{4}, y_{4}\right) \stackrel{\mu_{5} \mu_{6} \mu_{7} \mu_{8}}{\longleftrightarrow} \ldots .
\end{aligned}
$$

We obtain the following proposition from the definition of mutation (2.4).

Proposition 4.8 Consider the coefficient-free cluster algebra $\mathcal{A}\left(Q_{\mathrm{PVI}}, \boldsymbol{x}\right)$. For any $n \in \mathbb{Z}$, the cluster variables $w_{n}, x_{n}, W_{n}, X_{n}$ satisfy the bilinear equations:

$$
\begin{aligned}
& w_{n+2} X_{n}=x_{n+1} W_{n+1}+w_{n+1} X_{n+1}, \\
& x_{n+2} W_{n}=w_{n+1} X_{n+1}+x_{n+1} W_{n+1}, \\
& W_{n+2} x_{n}=X_{n+1} w_{n+1}+W_{n+1} x_{n+1}, \\
& X_{n+2} w_{n}=W_{n+1} x_{n+1}+X_{n+1} w_{n+1} .
\end{aligned}
$$

The bilinear equations (4.27) are obtained from the discrete mKdV equation (3.3) by imposing the reduction condition $w_{n+2}^{m-2}=w_{n}^{m}, x_{n+2}^{m-2}=x_{n}^{m}$ and $w_{n}:=w_{n}^{0}, x_{n}:=x_{n}^{0}, W_{n}:=w_{n-1}^{1}, X_{n}:=x_{n-1}^{1}$. This reduction of the difference equation corresponds to the $(2,-2)$-reduction of the dmKdV quiver. Moreover, the coefficients satisfy the $q$-Painlevé VI equation. 
Theorem 4.9 Consider the cluster algebra with coefficient $\mathcal{A}\left(Q_{\mathrm{PVI}}, \boldsymbol{x}, \boldsymbol{y}\right)$. For any $n \in \mathbb{Z}$, the coefficients $y_{n}, z_{n}, Y_{n}, Z_{n}$ satisfy the equations:

$$
\begin{aligned}
& y_{n+1} y_{n-1}=c_{2}^{-1} c_{3}^{2} c_{5}^{2} c_{1}^{2 n} \frac{\left(y_{n}+1\right)\left(c_{2} c_{3}^{-1} c_{4}^{(-1)^{n+1}} c_{5}^{-1} c_{6}^{(-1)^{n+1}} y_{n}+1\right)}{\left(y_{n}+c_{3} c_{4}^{(-1)^{n}} c_{1}^{n}\right)\left(y_{n}+c_{5} c_{6}^{(-1)^{n}} c_{1}^{n}\right)}, \\
& z_{n+1} z_{n-1}=c_{2} c_{3}^{2} c_{5}^{-2} c_{1}^{2 n} \frac{\left(z_{n}+1\right)\left(c_{3}^{-1} c_{4}^{(-1)^{n+1}} c_{5} c_{6}^{(-1)^{n}} z_{n}+1\right)}{\left(z_{n}+c_{3} c_{4}^{(-1)^{n}} c_{1}^{n}\right)\left(z_{n}+c_{2} c_{5}^{-1} c_{6}^{(-1)^{n+1}} c_{1}^{n}\right)}, \\
& Y_{n+1} Y_{n-1}=c_{2} c_{3}^{-2} c_{5}^{2} c_{1}^{2 n} \frac{\left(Y_{n}+1\right)\left(c_{3} c_{4}^{(-1)^{n}} c_{5}^{-1} c_{6}^{(-1)^{n+1}} Y_{n}+1\right)}{\left(Y_{n}+c_{2} c_{3}^{-1} c_{4}^{(-1)^{n+1}} c_{1}^{n}\right)\left(Y_{n}+c_{5} c_{6}^{(-1)^{n}} c_{1}^{n}\right)} \\
& Z_{n+1} Z_{n-1}=c_{2}^{3} c_{3}^{-2} c_{5}^{-2} c_{1}^{2 n} \frac{\left(Z_{n}+1\right)\left(c_{2}^{-1} c_{3} c_{4}^{(-1)^{n}} c_{5} c_{6}^{(-1)^{n}} Z_{n}+1\right)}{\left(Z_{n}+c_{2} c_{3}^{-1} c_{4}^{(-1)^{n+1}} c_{1}^{n}\right)\left(Z_{n}+c_{2} c_{5}^{-1} c_{6}^{(-1)^{n+1}} c_{1}^{n}\right)}
\end{aligned}
$$

where $c_{1}, c_{2}, \ldots, c_{6}$ are conserved quantities

$$
\begin{array}{ll}
c_{1}^{2}=\frac{y_{n+1} z_{n+1} Y_{n+1} Z_{n+1}}{y_{n} z_{n} Y_{n} Z_{n}}, & c_{2}=y_{n} z_{n} Y_{n} Z_{n} c_{1}^{-2 n}, \\
c_{3}^{2}=y_{2 n+1} z_{2 n+1} y_{2 n} z_{2 n} c_{1}^{-(4 n+1)}, & c_{4}^{2}=\frac{y_{2 n} z_{2 n}}{y_{2 n+1} z_{2 n+1}} c_{1}, \\
c_{5}^{2}=y_{2 n+1} Y_{2 n+1} y_{2 n} Y_{2 n} c_{1}^{-(4 n+1)}, & c_{6}^{2}=\frac{y_{2 n} Y_{2 n}}{y_{2 n+1} Y_{2 n+1}} c_{1} .
\end{array}
$$

If we put $f_{n}:=y_{2 n}, g_{n}:=y_{2 n+1}$, we obtain

$$
\begin{aligned}
& f_{n+1} f_{n}=c_{2}^{-1} c_{3}^{2} c_{5}^{2} c_{1}^{4 n+2} \frac{\left(g_{n}+1\right)\left(c_{2} c_{3}^{-1} c_{4} c_{5}^{-1} c_{6} g_{n}+1\right)}{\left(g_{n}+c_{3} c_{4}^{-1} c_{1}^{2 n+1}\right)\left(g_{n}+c_{5} c_{6}^{-1} c_{1}^{2 n+1}\right)}, \\
& g_{n} g_{n-1}=c_{2}^{-1} c_{3}^{2} c_{5}^{2} c_{1}^{4 n} \frac{\left(f_{n}+1\right)\left(c_{2} c_{3}^{-1} c_{4}^{-1} c_{5}^{-1} c_{6}^{-1} f_{n}+1\right)}{\left(f_{n}+c_{3} c_{4} c_{1}^{2 n}\right)\left(f_{n}+c_{5} c_{6} c_{1}^{2 n}\right)}
\end{aligned}
$$

from (4.20). These equations are nothing but the $q$-Painlevé VI equation 8 .

Proof By the definition of mutation (2.1), the coefficients $y_{n, m}, z_{n, m}, Y_{n, m}, Z_{n, m}$ satisfy

$$
\begin{aligned}
y_{n} & =y_{n, n-1}\left(y_{n-1}+1\right)\left(z_{n-1}^{-1}+1\right)^{-1}\left(Y_{n-1}^{-1}+1\right)^{-1}\left(Z_{n-1}+1\right), \\
y_{n, n-1} & =Z_{n-2}^{-1} \\
z_{n} & =z_{n, n-1}\left(z_{n-1}+1\right)\left(y_{n-1}^{-1}+1\right)^{-1}\left(Z_{n-1}^{-1}+1\right)^{-1}\left(Y_{n-1}+1\right), \\
z_{n, n-1} & =Y_{n-2}^{-1} \\
Y_{n} & =Y_{n, n-1}\left(Y_{n-1}+1\right)\left(Z_{n-1}^{-1}+1\right)^{-1}\left(y_{n-1}^{-1}+1\right)^{-1}\left(z_{n-1}+1\right), \\
Y_{n, n-1} & =z_{n-2}^{-1}, \\
Z_{n} & =Z_{n, n-1}\left(Z_{n-1}+1\right)\left(Y_{n-1}^{-1}+1\right)^{-1}\left(z_{n-1}^{-1}+1\right)^{-1}\left(y_{n-1}+1\right), \\
Z_{n, n-1} & =y_{n-2}^{-1}
\end{aligned}
$$

where we consider the index $n$ of the coefficients $y_{n, m}, z_{n, m}, Y_{n, m}, Z_{n, m}$ as $n \in \mathbb{Z} / 2 \mathbb{Z}$. The equations only 
for $y_{n}, z_{n}, Y_{n}, Z_{n}$ we obtain, are:

$$
\begin{aligned}
y_{n+2} & =\frac{\left(y_{n+1}+1\right)\left(Z_{n+1}+1\right)}{\left(z_{n+1}^{-1}+1\right)\left(Y_{n+1}^{-1}+1\right) Z_{n}}, \\
z_{n+2} & =\frac{\left(z_{n+1}+1\right)\left(Y_{n+1}+1\right)}{\left(y_{n+1}^{-1}+1\right)\left(Z_{n+1}^{-1}+1\right) Y_{n}}, \\
Y_{n+2} & =\frac{\left(Y_{n+1}+1\right)\left(z_{n+1}+1\right)}{\left(Z_{n+1}^{-1}+1\right)\left(y_{n+1}^{-1}+1\right) z_{n}}, \\
Z_{n+2} & =\frac{\left(Z_{n+1}+1\right)\left(y_{n+1}+1\right)}{\left(Y_{n+1}^{-1}+1\right)\left(z_{n+1}^{-1}+1\right) y_{n}} .
\end{aligned}
$$

We have

$$
\begin{aligned}
\frac{y_{n+2} z_{n+2} Y_{n+2} Z_{n+2}}{y_{n+1} z_{n+1} Y_{n+1} Z_{n+1}} & =\frac{y_{n+1} z_{n+1} Y_{n+1} Z_{n+1}}{y_{n} z_{n} Y_{n} Z_{n}} \\
y_{n+2} z_{n+2} Y_{n} Z_{n} & =y_{n+1} z_{n+1} Y_{n+1} Z_{n+1} \\
y_{n+2} Y_{n+2} z_{n} Z_{n} & =y_{n+1} z_{n+1} Y_{n+1} Z_{n+1}
\end{aligned}
$$

from $(4.32) \times(4.33) \times(4.34) \times(4.35),(4.32) \times(4.33)$, and (4.32) $\times(4.34$ ) respectively, and we obtain

$$
\frac{y_{n+1} z_{n+1} Y_{n+1} Z_{n+1}}{y_{n} z_{n} Y_{n} Z_{n}}=c_{1}^{2}
$$

from (4.36), where $c_{1}$ is a constant. In fact,

$$
y_{n} z_{n} Y_{n} Z_{n}=c_{2} c_{1}^{2 n}
$$

from $y_{n+1} z_{n+1} Y_{n+1} Z_{n+1}=c_{1}^{2} y_{n} z_{n} Y_{n} Z_{n}$, where $c_{2}$ is a constant. Eliminating $Y_{n}, Z_{n}$ from (4.37) and (4.40) we obtain $y_{n+2} z_{n+2}=c_{1}^{2} y_{n} z_{n}$. If we eliminate $z_{n}, Z_{n}$ from (4.38) and (4.40) we obtain $y_{n+2} Y_{n+2}=$ $c_{1}^{2} y_{n} Y_{n}$. Therefore,

$$
y_{n} z_{n}=c_{3} c_{4}^{(-1)^{n}} c_{1}^{n}, \quad y_{n} Y_{n}=c_{5} c_{6}^{(-1)^{n}} c_{1}^{n},
$$

where $c_{3}, c_{4}, c_{5}, c_{6}$ are constants. Eliminating $z_{n}, Y_{n}$ from (4.40) and (4.41) we obtain

$$
y_{n}=c_{2}^{-1} c_{3} c_{4}^{(-1)^{n}} c_{5} c_{6}^{(-1)^{n}} Z_{n} .
$$

Finally, eliminating $z_{n}, Y_{n}, Z_{n}$ from (4.41), (4.42), and (4.32) we obtain the q-Painlevé VI equation (4.28). The equations for $z_{n}, Y_{n}, Z_{n}$ are the same as that for $y_{n}$.

\section{Conclusion}

We have shown that cluster variables can satisfy the discrete KdV equation, the Hirota-Miwa equation, the discrete $\mathrm{mKdV}$ equation, and the discrete Toda equation, if we take appropriate quivers of initial seeds. We have also shown that the coefficients of certain cluster algebras satisfy the $q$-Painlevé I,II,III,VI equations. These cluster algebras are obtained from a reduction of the quivers for some integrable partial difference equations. The $q$-Painlevé III equation (4.20) and the $q$-Painlevé VI equation (4.28) are classified as type $\left(A_{2}+A_{1}\right)^{(1)}$ and $D_{5}^{(1)}$ in the classification of root systems [8]. So far we have not obtained the $q$-discrete Painlevé equations of type $A_{4}^{(1)}$ and $E_{n}^{(1)}$ from cluster algebras. In the future, we wish to clarify the relations between these equations and cluster algebras. Quivers of higher order analogue of $q$-Painlevé I,II equations are obtained in [9]. To obtain quivers of higher order analogues of the $q$-Painlevé III,VI equations is also a problem we wish to address in the future.

\section{References}

[1] S. Fomin and A. Zelevinsky, "Cluster algebras IV: Coefficients", Compositio Mathematica 143, (2007), 112-164. 
[2] S. Fomin, "Total positivity and cluster algebras", Proceedings of the International Congress of Mathematicians, 2, (2010), 125-145.

[3] A. P. Fordy and R. J. Marsh, "Cluster mutation-periodic quivers and associated Laurent sequences", Journal of Algebraic Combinatorics, 34, no. 1, (2011), 19-66.

[4] R. Hirota, "Nonlinear Partial Difference Equations. I. A Difference Analogue of the Korteweg-de Vries Equation", Journal of the Physical Society of Japan, 43, (1977), 1424-1433.

[5] T. Miwa, "On Hirota's difference equations", Proceedings of the Japan Academy, Series A, Mathematical Sciences, 58, no. 1, (1982), 9-12.

[6] N. Okubo, "Discrete integrable systems and cluster algebras", RIMS Kokyuroku Bessatsu, Research Institute for Mathematical Sciences, B41, (2013), 25-42.

[7] R. Hirota, "Discrete Analogue of a Generalized Toda Equation", JPSJ, 50, (1981), 3785-3791.

[8] H. Sakai, "Rational surfaces associated with affine root systems and geometry of the Painlevé equations", Communications in Mathematical Physics, 220, (2001), 165-229.

[9] A. N. W. Hone and R. Inoue, "Discrete Painlevé equations from Y-systems", J. Phys. A: Math. Theor., 47, 474007, (2014).

[10] B. Grammaticos and A. Ramani, "The hunting for the discrete Painlevé equations", Reg. and Chaot. Dyn. , 5, (2000), 53-66.

[11] M. D. Kruskal, K. M. Tamizhmani, B. Grammaticos and A. Ramani, "Asymmetric discrete Painlevé equations", Reg. Chaot. Dyn. 5, No. 3, (2000), 273-280.

[12] K. Kajiwara, K. Kimura, "On a $q$-difference Painlevé III equation: I. Derivation, symmetry and Riccati type solutions", J. Nonlin. Math. Phys. , 10, (2003), 86-102. 